ESAIM: PROCEEDINGS AND SURVEYS, May 2021, Vol. 70, p. 14-30

Arnaud Duran, Benoît Fabrèges, Pauline Lafitte, Frédéric Lagoutière, Fabien Marche, Frédéric Rousset

\title{
COMPARISON OF MODELS FOR THE SIMULATION OF LANDSLIDE GENERATED TSUNAMIS
}

\author{
E. Audusse ${ }^{1}$, J.G. Caldas Steinstraesser ${ }^{2}$, L. Emerald ${ }^{3}$, P. Heinrich ${ }^{4}$, A. \\ PARIS $^{4,5}$ AND M. PARISOT ${ }^{6}$
}

\begin{abstract}
In this paper, we analyze the relevance of the use of the shallow water model and the Boussinesq model to simulate tsunamis generated by a landslide. In a first part, we determine if the two models are able to reproduce waves generated by a landslide. Each model has drawbacks but it seems that it is possible to use them together to improve the simulations. In a second part we try to recover the landslide displacement from the generated wave. This problem is formulated as a minimization problem and we limit the number of parameters to determine assuming that the bottom can be well described by an empirical law.

Résumé. Dans cet article, nous analysons la pertinence de l'utilisation de modèles intégrés suivant la verticale tels que le modèle de Saint-Venant et le modèle de Boussinesq pour simuler les tsunamis générés par un glissement de terrain. Dans une première partie, nous déterminons si les deux modèles sont capables de reproduire les vagues générées par un glissement de terrain. Chaque modèle présente des inconvénients mais il semble qu'il soit possible de les utiliser ensemble pour améliorer les simulations. Dans une deuxième partie, nous essayons de retrouver le déplacement du glissement de terrain à partir de la vague générée. Ce problème a été formulé comme un problème de minimisation et nous limitons le nombre de paramètres à déterminer en supposant que le fond peut être bien décrit par une loi empirique.
\end{abstract}

\section{INTRODUCTION}

This work is devoted to the numerical comparison of three different fluid mechanics models in simulating landslide tsunamis: the Navier-Stokes model, the Boussinesq model and the shallow water model. The former is considered here as a reference since the two latter are simplifications of it. However, its high computational cost and potential numerical difficulties, such as an excessive numerical diffusion, the definition of boundaries conditions or reflections at the boundaries, motivate the use of simplified models and make the computed solution not always clearly closer to the reality for some applications such as the simulation of tsunamis because of the large space scale involved. The simplified models are based on a depth-averaging. For these reasons simulations are much less expensive than with those solving the Navier-Stokes model. The shallow water model neglects the

\footnotetext{
${ }^{1}$ Université Paris 13, Laboratoire d'Analyse, Géométrie et Applications, 99 av. J.-B. Clément, F-93430 Villetaneuse, France

2 Team LEMON, INRIA Sophia Antipolis - Méditerrannée, Bât 5 - CC05 017, 860 rue Saint-Priest, 34095 Montpellier Cedex 5, France. Univ. Montpellier, CNRS, IMAG, UMR 5149, 34090 Montpellier, France

${ }^{3}$ Université de Rennes 1, IRMAR, 35042 Rennes CEDEX France

4 CEA, DAM, DIF, 91297 Arpajon Cedex

${ }^{5}$ Université de Pau et des Pays de l'Adour, E2S UPPA, SIAME, Anglet, France

6 Team CARDAMOM, INRIA Bordeaux Sud Ouest, 200 Avenue de la Vieille Tour, 33405 Talence cedex, France Univ. Bordeaux, CNRS, Bordeaux INP, IMB, UMR 5251, F-33400, Talence, France

(C) EDP Sciences, SMAI 2021
}

This is an Open Access article distributed under the terms of the Creative Commons Attribution License (http://creativecommons.org/licenses/by/4.0), which permits unrestricted use, distribution, and reproduction in any medium, provided the original work is properly cited. 


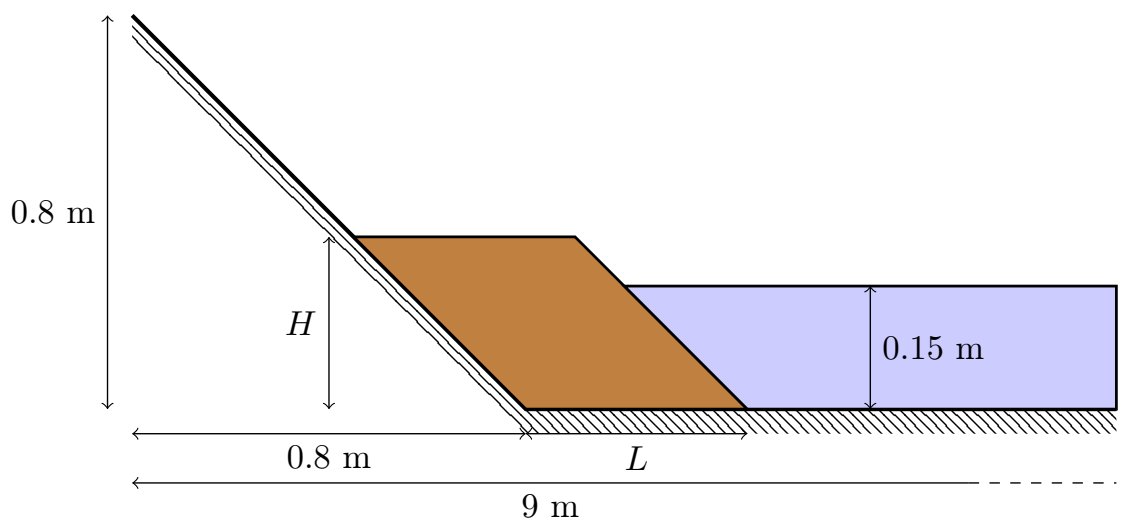

Figure 1. Initial state of the test case. The landslide is in brown and the water is in blue.

dispersive effects by assuming a hydrostatic pressure. The Boussinesq model takes into account the dispersive effects assuming small perturbation from a main water depth, but it is based on a non-conservative form that can leads to wrong solutions in the case of strongly variations of the free surface. A deeper description of the models is done in $\$ 2.2$ as well as a description of the numerical strategy used to simulate them.

The depth-averaging models are largely used in the simulation of tsunamis 1 - 8 . In particular, the shallow water model is able to predict with a good accuracy the arrival time on the coast of the tsunami. However the amplitude of the wave is not well predict by the shallow water model, in particular second and third waves which are sometimes more destructive than the first despite they are smaller. The Boussinesq model leads to a better description of both the wave slope and the run-up area.

We address the question of the numerical strategy to recover the tsunami waves generated by landslides. This question is not trivial at the scale of the application and several strategies exist in the literature [9, 10]. We propose to explore the possibility to recover the bottom displacement from the free surface elevation using a inverse problem of a simplified model. This strategy assumes two technical steps that have to be first validated and this is the aim of this proceeding. In a first part \$3, we investigate the ability of the simplified models to reproduce the wave obtained by the reference model (Navier-Stokes simulated by OpenFOAM). The important question lies on the strategy used to represent the landslide and in the current work we propose to recover the bottom displacement from the result of the reference model. The drawback of this strategy is that in practice, we do not want to simulate the reference model at the scale of the application. In the second part $\$ 4$, we investigate the possibility of recovering the bottom displacement from the free surface elevation at given positions. Inverse problems of models that develop discontinuous solutions are generally complex, see [11]. Nevertheless, this preliminary study tries to identify the main practical difficulties.

\section{Description OF THE STUdY}

\subsection{The test case}

The test case we based our study on is inspired from the literature, in particular [12. It is a 2D test case, where $x \in \mathbb{R}$ is the horizontal coordinate and $z \in \mathbb{R}$ is the vertical coordinate, on an idealized bathymetry, called hereafter the substratum, defined by

$$
B(x)=\max (0, D-x)-D
$$

with the offshore depth $D=0.15 \mathrm{~m}$. The time coordinate will be denoted by $t \in \mathbb{R}_{+}$. At the initial state, the landslide is at rest and has the shape of a parallelogram of vertices $\left(x_{0},-D\right),\left(x_{0}+L,-D\right),\left(x_{0}+L-H, H-D\right)$ 
and $\left(x_{0}-H, H-D\right)$. Several values of the height $H$ and the length $L$ will be explored in the study. The water is initially at rest with a flat free surface at $z=0 \mathrm{~m}$. The initial state is illustrated on Figure 1 .

The boundary conditions depend on the model and are described in $\$ 2.2$.

\subsection{The models and their numerical resolution}

The numerical simulations of the landslide-generated waves were performed considering three models: the Navier-Stokes model, the Boussinesq model and the shallow water model. Because of their different mathematical properties, they are solved using different numerical tools presented in what follows. Note that the numerical strategies presented for each model are not the only ones existing in the literature. They have advantages as well as drawbacks that we try to summarize in this subsection.

For the sake of simplicity, we do not consider the second horizontal direction $y$ since our test case does not depend on it. Note however that the models as well as the numerical tools used in this work can take it into account, (i.e. 3D $(x, y, z)$ simulations for the Navier-Stokes model and 2D $(x, y)$ simulations for the shallow water model and the Boussinesq model).

\subsubsection{OpenFOAM solver of the free surface incompressible Navier-Stokes model}

The fluid dynamics for macroscopic applications are classically described by the incompressible Navier-Stokes equations. However, the modeling of tsunamis generated by landslide also requires the description of the water surface, also called free surface, and the description of the dynamics of the landslide. In this work we propose to adopt the VOF (Volume Of Fluid) strategy, i.e. globally describes the flow solving the incompressible Navier-Stokes equations in the whole computational domain with physical parameters such as density and viscosity depending on the fluid locally present. More precisely, the fluid is identified using the color function $\phi(t, x, z) \in[0,2]$ satisfying the following advection equation

$$
\partial_{t} \phi+u \cdot \nabla \phi=0
$$

where $u(t, x, z) \in \mathbb{R}^{2}$ is the velocity of the fluid. When $\phi=0, \phi=1$ and $\phi=2$ we consider that the fluid is respectively the air, the water and the landslide. The velocity satisfies the Navier-Stokes equations

$$
\rho\left(\partial_{t} u+u \cdot \nabla u\right)=-\nabla p+\nabla \cdot(\mu \nabla u)+\rho g e_{z}
$$

where $e_{z}$ is the unit downward vector, $g$ is the gravitational acceleration, $\rho(\phi) \in \mathbb{R}_{+}$is the density of the fluid and $\mu(\phi) \in \mathbb{R}_{+}$its dynamic viscosity. These two parameters are piecewise linear reconstructions depending on the color function, i.e.

$$
\kappa(\phi)= \begin{cases}(1-\phi) \kappa_{0}+\phi \kappa_{1} & \text { if } 0 \leq \phi \leq 1 \\ (2-\phi) \kappa_{1}+(\phi-1) \kappa_{2} & \text { if } 1<\phi \leq 2\end{cases}
$$

where $\kappa$ stands for $\rho$ and $\mu$ and the parameter $\rho_{0}$ (resp. $\mu_{0}$ ) stands for the density (resp. viscosity) of the air, $\rho_{1}$ (resp. $\mu_{1}$ ) stands for the density (resp. viscosity) of the water, and $\rho_{2}$ (resp. $\mu_{2}$ ) stands for the density (resp. viscosity) of the landslide. In this study we set these parameters close to the physical values, i.e. $\rho_{0}=1 \mathrm{~kg} \cdot \mathrm{m}^{3}$, $\rho_{1}=1000 \mathrm{~kg} \cdot \mathrm{m}^{3}, \rho_{2}=1500 \mathrm{~kg} \cdot \mathrm{m}^{3}, \mu_{0}=1.48 \cdot 10^{-5} \mathrm{~Pa} \cdot \mathrm{s}, \mu_{1}=10^{-3} \mathrm{~Pa} \cdot \mathrm{s}, \mu_{2}=2.222 \mathrm{~Pa} \cdot \mathrm{s}$. The pressure $p(t, x)$ is such that the flow satisfies the divergence free condition, i.e.

$$
\nabla \cdot u=0
$$

The set of equations is completed by an initial condition and the boundary conditions described in the test case, see 2.1 .

The numerical approximation of the Navier-Stokes model is realized using the module multiphaseInterFoam of the $\mathrm{C}++$ open source software OpenFOAM (Open-source Field Operation And Manipulation) [13. 
We assume a slip boundary condition at the substratum $z=B(x)$, i.e. $u \cdot \nabla B=0$. The friction at the substratum is neglected to limit the difference between the models. However, it is not always clear how to translate a no-slip boundary condition or a Navier Boundary condition from the Navier-Stokes model to the shallow water model or the Boussinesq model. For computational reasons, we need to introduce a top boundary condition, set at $z=0.65 \mathrm{~m}$, and a left and right boundary conditions, set respectively at $x=-0.65 \mathrm{~m}$ and $x=8.35 \mathrm{~m}$. All of them are defined as open boundary conditions. The space step is set in the two directions to $5 \cdot 10^{-3} \mathrm{~m}$ in order to limit the numerical diffusion. But it makes the computation time fast enough to allow several sizes of landslides. The time step used for the computations is set to $10^{-3} \mathrm{~s}$ whereas the frequency of the output is set to $2 \cdot 10^{-2} \mathrm{~s}$.

\subsubsection{Hydra solver of the shallow water model}

The shallow water model is one of the simplest for hydrology. Its well understood mathematical structure allowed very efficient and robust numerical computation. It can be derived from the multiphase Navier-Stokes model (2)-(5) integrating only the water phase, i.e. $\phi=(1,0)$, for an irrotational flow and under a long-wave assumption [14]. The classical form of the model reads

$$
\begin{aligned}
\partial_{t} \bar{h}+\partial_{x}(\bar{h} \bar{u}) & =0 \\
\partial_{t}(\bar{h} \bar{u})+\partial_{x}\left(\bar{h} \bar{u}^{2}+\frac{g}{2} \bar{h}^{2}\right) & =g \bar{h} \partial_{x} d
\end{aligned}
$$

where $\bar{h}(t, x)$ is the water depth and $\bar{u}(t, x)$ is the main horizontal velocity. The bottom elevation $d(t, x)$ is an input of the model and we discuss further 3.1 .1 the strategy to obtain it.

The shallow water model is solved using the numerical tools Hydra that is based on a finite volume Godunovtype solver with MUSCL second order reconstruction and a second order Runge-Kutta time scheme [15. The bathymetry source term is treated using the hydrostatic reconstruction [16.

The left and right boundaries are set respectively at $x=-0.65 \mathrm{~m}$ and $x=8.35 \mathrm{~m}$ and open boundary conditions are used. Note that the open boundary condition is not treated in the same way by the different models. The horizontal space domain was discretized with an homogeneous grid of space step $5 \cdot 10^{-3} \mathrm{~m}$. We used an adaptive time step based on the CFL condition with the CFL parameter set to 0.9. However, the outputs are separated in time by $0.1 \mathrm{~s}$.

\subsubsection{AVALANCHE solver of the Boussinesq model}

The Boussinesq model is largely used for the propagation of waves in ocean modeling. It can be derived from the multiphase Navier-Stokes model (2)-(5) by integrating only the water phase, i.e. $\phi=(1,0)$, for an irrotational flow, weakly nonlinear and weakly dispersive [14]. The Boussinesq model can be written under the form 17$]$

$$
\begin{aligned}
\partial_{t} \underline{\eta}+\partial_{x}(\underline{h u}) & =-\partial_{t} d \\
\partial_{t} \underline{u}+\underline{u} \partial_{x} \underline{u} & =-g \partial_{x} \underline{\eta}+\frac{d^{3}}{3} \partial_{x}^{2}\left(\partial_{t} \underline{u}\right)+\frac{d}{2} \partial_{t} \underline{u} \partial_{x}^{2} \underline{u}+d \partial_{x} d \partial_{x}\left(\partial_{t} \underline{u}\right)
\end{aligned}
$$

where $\eta(t, x)$ is the free surface elevation, $\underline{h}(t, x)=\eta+d$ is the water depth, $\underline{u}(t, x)$ is the main horizontal velocity. As for the shallow water model, the bottom elevation $d(t, x)$ is an input of the model.

The Boussinesq model is solved using the numerical tools AVALANCHE developed at the CEA to simulate tsunamis (second order finite difference methods with Crank-Nicolson time scheme). It was successfully used to study subaerial or submarine landslide tsunamis [1-7].

As well as in the case of the shallow water model, the friction is neglected and open boundary conditions are considered at the left $(x=-0.65 \mathrm{~m})$ and right $(x=8.35 \mathrm{~m})$ boundaries. We use the same space step of $5 \cdot 10^{-3}$ $\mathrm{m}$, an adaptive time step based on the CFL condition with the CFL parameter set to 0.9 and the outputs are separated in time by $0.1 \mathrm{~s}$. 


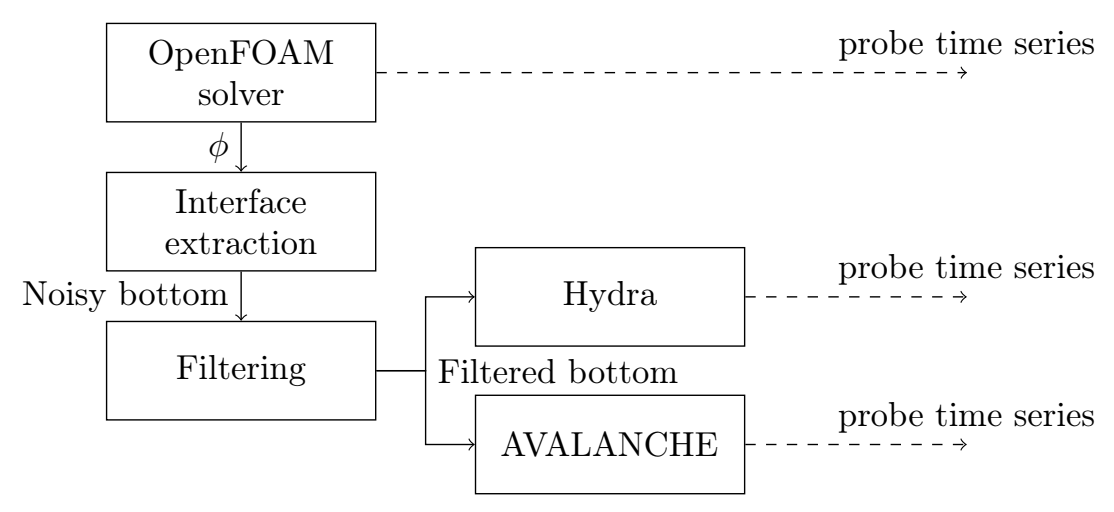

FigURE 2. Strategy of computation.

\section{Comparison OF MOdels}

\subsection{The methodology}

From the description of the models $\$ 2.2$ an important difference can be noticed by the fact that the simplified models (6) and (7) do not describe the dynamic of the landslide but only the dynamic of the water. Several strategies exist in the literature to model landslide using the same kind of simplified model [18, 19]. However the difference of the dynamic of the landslide obtained by this strategy compared to the result of the multiphase Navier-Stokes model (2)-(5) will make the comparison of the free surface unclear. Since our study focus on the waves generation, we adopt another strategy where the efficient bottom of the simplified model is not the substratum given in (1) but is obtained by post-treatment of the OpenFOAM Navier-Stokes results, see $\$ 3.1 .1$

We would like to point out another major difference to keep in mind in the comparison of the models. The friction in the simplified models is neglected. This assumption is consistent with the slip condition at the substratum in the simulation of the Navier-Stokes model. However the bottom elevation is located at the interface between the landslide and the water for the simplified models. Because of the viscosity of the landslide, a friction should be considered when the landslide thickness does not vanish. Nevertheless, this value is very difficult to identify and we assumed it small enough to be neglected.

\subsubsection{Strategy of computation of the bottom displacement}

In the current section we detail the computational strategy used to define the bottom elevation in the simplified models (6) and (7). It is illustrated in Figure 2

First of all, the multiphase Navier-Stokes model (2)-(5) is solved using OpenFOAM.

For each output of this computation, we extracted the interface between the landlside and the water, defined here as the surface satisfying $\phi=1.5$, to be used as the bottom elevation for the simplified model (6) and (7). The interface extraction was realized using the software ParaView that allowed to obtain series of points on each surface where it crosses the mesh of the computation, so not regularly spaced. These series presented some oscillations, probably coming from the VOF strategy, and were polluted by some points at the substratum $z=B(x)$, in particular when the landslide becomes thin.

To clean these files we applied a filter that first removed the points too close to the substratum, i.e. $z<B(x)+5 \cdot 10^{-3}$, then we applied a Laplacian filter to smooth the angular regions. Eventually we interpolated (linear interpolation with the left and right closer points) the series on the mesh of the simplified models. We obtained a description of the bottom elevation on the mesh of the simplified models at each output time of the OpenFOAM simulation, see Figure 3. However, because of the adaptive time step strategy, we needed to interpolate these files during the computation to obtain the bottom elevation at each time step of Hydra and 


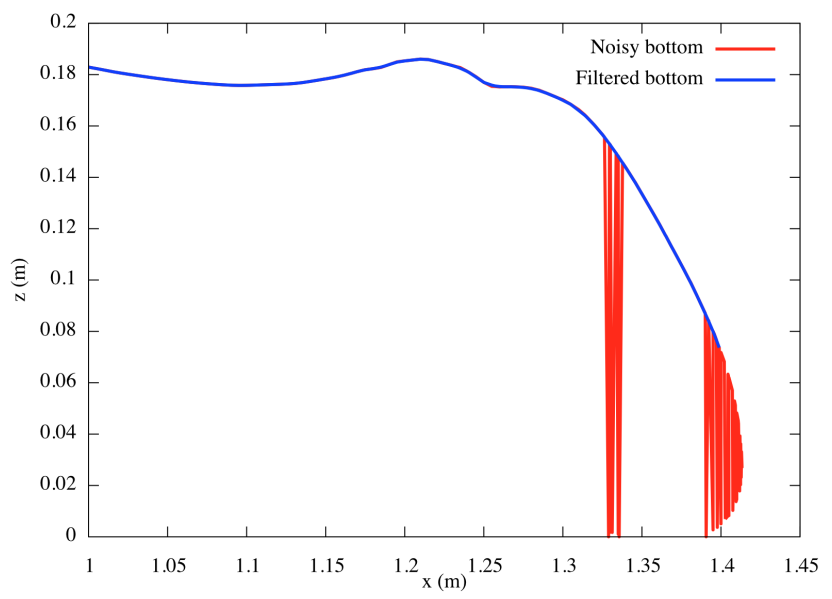

FiguRE 3. Illustration of the filtering process. Red line is the bottom after the extraction step. Blue line is the bottom after the filtering step.

AVALANCHE. Once again, a linear interpolation between the closest past and future filtered bottom was used.

\subsubsection{Simulated data based and treatment}

Several simulations were performed using the strategy described in 3.1 .1 by varying the shape of the initial landslide block using the height $H$ (between 0.2 and $0.5 \mathrm{~m}$ every $0.05 \mathrm{~m}$ ) and the length $L$ (between 0.1 and 0.6 m every $0.05 \mathrm{~m}$ ), see Figure 1. For each simulation, we compared the time series of the free surface at some gauges. This strategy follows the experimental process in which the free surface elevation is usually recovered only by some buoys at given positions.

In a first time, we compare qualitatively the results of the different models. Our first observation is that there are two distinct regions. In the area where the landslide is moving, the predominant physical phenomenon is the generation of the wave due to the variation of the bottom. Hereafter, we call this region the wave generation zone. The comparisons in this zone are described in 3.2 .1 . Far from the landslide, the dynamic of the wave is essentially a propagation phenomenon. Consequently, we call this region the wave propagation zone. The comparisons in this zone are described in $\$ 3.2 .2$.

Obviously the most important region is at the transition between the two zones defined above, around the place that the landslide finally reaches. In order to realize statistical analysis, we would like to compare the flow at a unique position, ideally in the transition area. However, it is not obvious how to clearly determine this point and it seems relevant to not change the position of the probe depending on the landslide. Hopefully, for a large majority of the simulated landslide, the right bound of the wave generation zone is close to $x=1.15 \mathrm{~m}$. So the probe $x=1.35 \mathrm{~m}$ seems very relevant because it is located just after the place where the landslide stops for almost all the simulations. More precisely, among the 77 simulations we ran, 61 were used to identify quantities of interest to characterize the generated waves, see 3.2 .3 . In the other simulations, the landslide reaches the probe $x=1.35 \mathrm{~m}$, completely changing the results. We also highlight the regime of validity of the simplified models.

Eventually, in 3.2 .4 , we propose a numerical strategy trying to combine the best of both simplified models by using one or another depending on the position: we use the shallow water model $(6)$ in the wave generation zone and the Boussinesq model (7) in the wave propagation zone. This domain decomposition strategy is well known and already used in the case of breaking waves, where the Boussinesq model is solved in the propagation zone and the shallow water model in the surf zone 20 . 

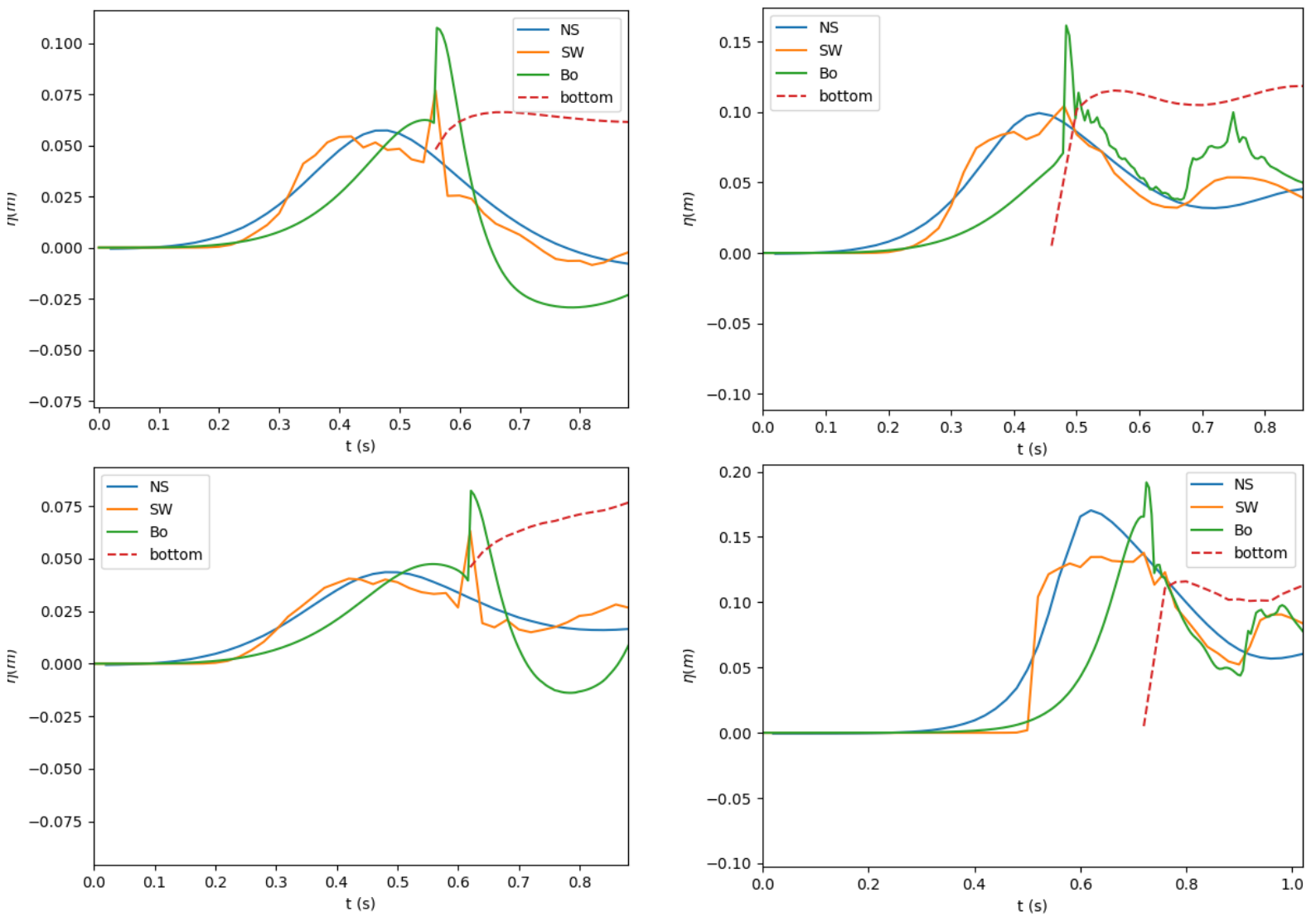

Figure 4. Comparison between the results of the three models for four selected test cases, in the wave generation zone. The top left corresponds to the case i) at the probe $x=0.55 \mathrm{~m}$. The top right corresponds to the case ii) at the probe $x=0.75 \mathrm{~m}$. The bottom left corresponds to the case iii) at the probe $x=0.95 \mathrm{~m}$. The bottom right corresponds to the case iv at the probe $x=1.15 \mathrm{~m}$. Blue, orange and green lines represent respectively the Navier-Stokes, the shallow water and the Boussinesq simulations. Red dashed line represents the evolution of the bottom.

\subsection{The numerical results}

In the two first parts, we focus on four selected cases:

i) $(H, L)=(0.25 \mathrm{~m}, 0.2 \mathrm{~m})$,

ii) $(H, L)=(0.3 \mathrm{~m}, 0.4 \mathrm{~m})$,

iii) $(H, L)=(0.2 \mathrm{~m}, 0.6 \mathrm{~m})$,

iv) $(H, L)=(0.45 \mathrm{~m}, 0.4 \mathrm{~m})$.

\subsubsection{Comparison in the wave generation zone}

Figure 4 represents the time series of the water surface elevation for the four test cases at gauge located at the border of their wave generation zone, i.e. case i) $x=0.55 \mathrm{~m}$, case iii) $x=0.75 \mathrm{~m}$, case iii) $x=0.95 \mathrm{~m}$ and case iv $x=1.15 \mathrm{~m}$. In the same graphs we plot the landslide thickness (red dashed line) when the landslide reaches the position of the probe.

We first observe that both simplified models, for which the dynamic of the landslide was imposed as a given bathymetry, are very sensitive to the variation of the bathymetry. Indeed, in the beginning of the red dashed 
line in each graph in Figure 4, we can observe the formation of peaks for the shallow water and the Boussinesq simulations. These peaks seem artificial, due to our strategy of imposing the bottom displacement and the fact that we do not have a proper energy closure. Studies in the literature that impose the landslide displacement using the same kind of simplified model (for example [18,19]) do not present such kind of peaks. However the dynamic of the landslide is significantly different.

We also observe that the shallow water model (6) presents qualitatively better results than the Boussinesq model (7) in the wave generation zone. This behavior was observed for all the test cases performed. Our explanation of this observation is that the Boussinesq model is weakly nonlinear. It means the water elevation in the dispersive part of the model (RHS of the momentum equation) was replaced by the mean depth $d$. However in the derivation of the model, the mean depth should not change in time, or at least much slower than the free surface, which is not the case in our test case. In addition, we can remark that the advection term of the Boussinesq model (LHS of the momentum equation) is written in the non-conservative form. It is well known that this form is not suitable for discontinuous solutions, at least in the case of the shallow water model, because it leads to wrong weak solutions. It is generally not a problem for the Boussinesq model, which does not develop discontinuous solutions. However, in our test case, because of the discontinuous bottom, discontinuous solutions appear.

\subsubsection{Comparison in the wave propagation zone}

Here we focus on the wave propagation zone for the same test cases as in $\$ 3.2 .1$. The time series of the water surface elevation at the gauges case ii $x=1.35 \mathrm{~m}$, case iii) $x=1.55 \mathrm{~m}$, case iii) $x=1.75 \mathrm{~m}$ and case iv $x=1.95$ $\mathrm{m}$ are represented in Figure 5 By definition of the wave propagation zone, the bottom does not reach the gauges.

We remark that in the wave propagation zone, the shallow water is not always the best model in recovering the results of the Navier-Stokes model. Moreover the results differ strongly from one test case to another. While for the case ip and case iii) the Boussinesq model is better, for the case iv it is the shallow water model. Finally, for the case iii) both models seem quite far from the Navier-Stokes solution.

In the simulations realized in this work, the Boussinesq model behaves better in the wave propagation zone for small landslides, while the shallow water model behaves better for big landslides. One can explain this by the fact that the Boussinesq model is a better model for the wave propagation since it takes into account the dispersive effects. However, for big landslides and since the interactions with the moving bathymetry is worse than in the case of the shallow water model, the error created during the wave generation cannot be compensated in the propagation zone. Anyway this observation suggests that the quality of the approximations given by the simplified models is related to the volume of the landslide. However the results of case iii) and case iii) are too different in spite of the same volume of the landslide. In Figure 6, we show two simulations with the same volume of landslide leading to different conclusions. The graphs on the top, for parameters $(H, L)=(0.25 \mathrm{~m}, 0.45$ $\mathrm{m})$, show better approximations with the shallow water model in the wave generation zone but worse than the Boussinesq model in the propagation zone. The graphs on the bottom, for parameters $(H, L)=(0.45 \mathrm{~m}, 0.25 \mathrm{~m})$, show better approximations with the shallow water model in both zones, generation and propagation. In the next section, we investigate a better criterion than the volume of the landslide to characterize the qualitative properties of the two simplified models in matching the Navier-Stokes solutions.

For now, we may conclude that the volume of the landslide is not the most determining criterium for the behavior of the shallow water model and the Boussinesq model. Instead, we can suppose that there exists a more direct link with the energy transferred from the landslide to the water. However, in practice it is not easy to measure aprior $i$ the energy transferred. We first show in Figure 7 that the energy transferred from the landslide to the water is strongly related to the initial energy of the landslide. More precisely, for all the simulations we performed, between $50 \%$ and $60 \%$ of the initial energy was transferred to the water. Let us detail how we compute the energies. Since the flow is initially at rest, the initial energy of the landslide can be computed from its initial configuration, i.e. $\mathcal{P}^{0}=\mathcal{P}(t=0)$ where $\mathcal{P}$ is the potential energy given by

$$
\mathcal{P}(t)=\frac{g}{2} \int_{x}(\beta(t, x)-B(x))^{2} \mathrm{~d} x
$$



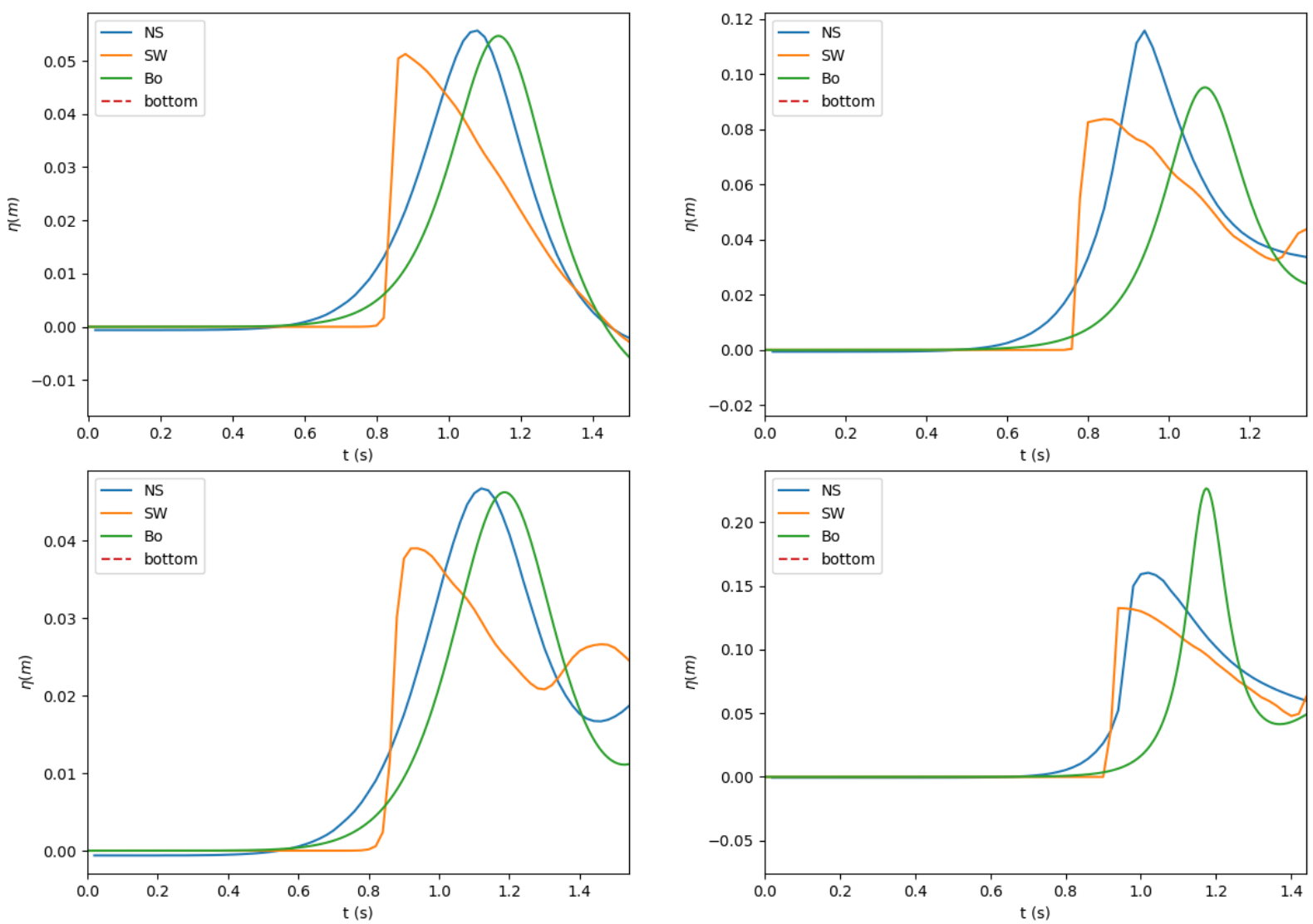

Figure 5. Comparison between the results of the three models for four selected test cases, in the wave generation zone. The top left corresponds to the case i at the probe $x=1.35 \mathrm{~m}$. The top right corresponds to the case ii) at the probe $x=1.55 \mathrm{~m}$. The bottom left corresponds to the case iii) at the probe $x=1.75 \mathrm{~m}$. The bottom right corresponds to the case iv at the probe $x=1.95 \mathrm{~m}$. Blue, orange and green lines represent respectively the Navier-Stokes, the shallow water and the Boussinesq simulations.

where $\beta(t, x)$ is the top interface of the landslide, i.e. $\phi=1.5$. This integral is computed numerically from the output of OpenFOAM. Instead of the energy transferred, we can easily compute the final potential energy, i.e. $\mathcal{P}(t=2)$. At time $t=2 \mathrm{~s}$ the landslide is at rest so all its energy is potential energy. By subtracting the two potential energies we obtain the total energy lost by the landslide during its motion. In fact, it is not exactly the energy transferred to the water (even if we neglect numerical artifices) because of the landslide's viscosity. However, once again we assume that the energy lost by viscosity effects is negligible.

\subsubsection{Quantities of interest and correlations between the models}

The correlations between some physical quantities were investigated in the probe located at the beginning of the wave propagation zone, i.e. $x=1.35 \mathrm{~m}$. In case of linear correlation, we indicate on the graphs the coefficient of correlation $R^{2}$. As the extent of the wave generation zone is not the same for all the tests, the ones corresponding to the biggest amounts of landslide were not considered when computing the correlation curves presented here, since the selected gauges are in their wave generation zone, affecting the quantities considered. Therefore, among the 77 test cases performed, 61 were retained for the correlations. 

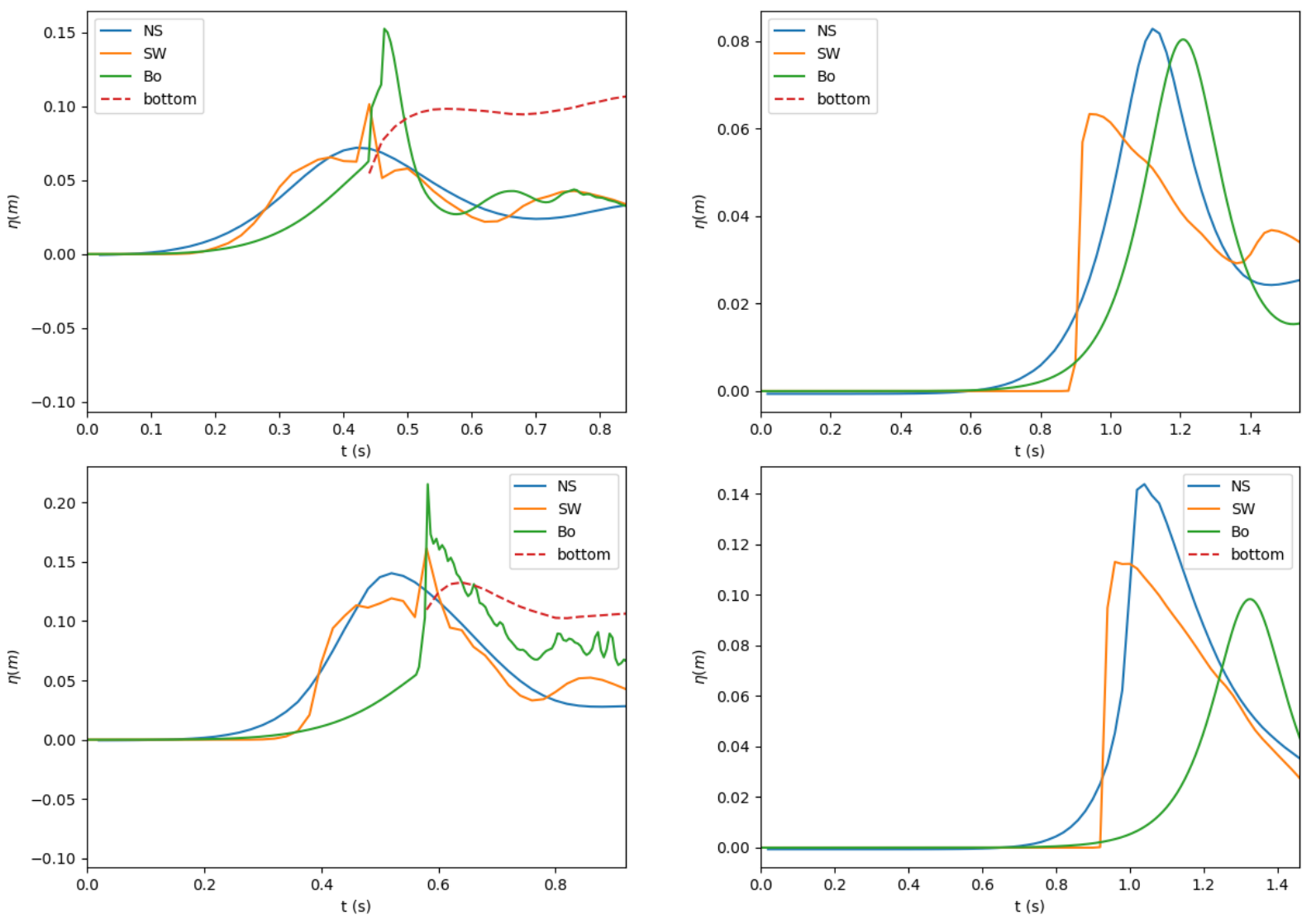

Figure 6. Comparison between the results of the three models with the same landslide size. The top line corresponds to the parameters $(H, L)=(0.25 \mathrm{~m}, 0.45 \mathrm{~m})$ while the bottom line corresponds to the parameters $(H, L)=(0.45 \mathrm{~m}, 0.25 \mathrm{~m})$. The left column corresponds to a probe at $x=0.75 \mathrm{~m}$ (wave generation zone) while the right column corresponds to a probe at $x=1.75 \mathrm{~m}$ (wave propagation zone).

First, we confirm our observation that the volume of the landslide does not characterize well the wave generated. In Figure 8, the left graph shows the correlation between the amplitude of the wave generated by the landslide and the volume of the landslide. It is clear that an information is missing. The right graph illustrates the correlation between the amplitude and the energy transferred to the water. We observe that almost all the simulations can be fitted by a logarithm law.

Now we compare the models in function of the energy transferred to the water. Figure 9 shows the deviation of the first wave at the chosen probe in terms of time delay (left column) and in terms of amplitude (right column) of the shallow water model (first line) and the Boussinesq model (second line). This result confirms the first observations, that the shallow water model seems to give better approximation for waves generated by important landslides (in terms of energy) while the Boussinesq model leads to better approximations for small landslides (see 3.2 .1 and $\$ 3.2 .2$. In addition, we observe that the shallow water model is always late compared to the reference Navier-Stokes solution while the Boussinesq model is ahead. The results in terms of difference of amplitude are not as clear. For the shallow water model, a logarithm deviation seems to appear. However, the deviation of the Boussinesq model appears as a succession of logarithm that seems to indicate that some informations are still missing to characterize the flow. 


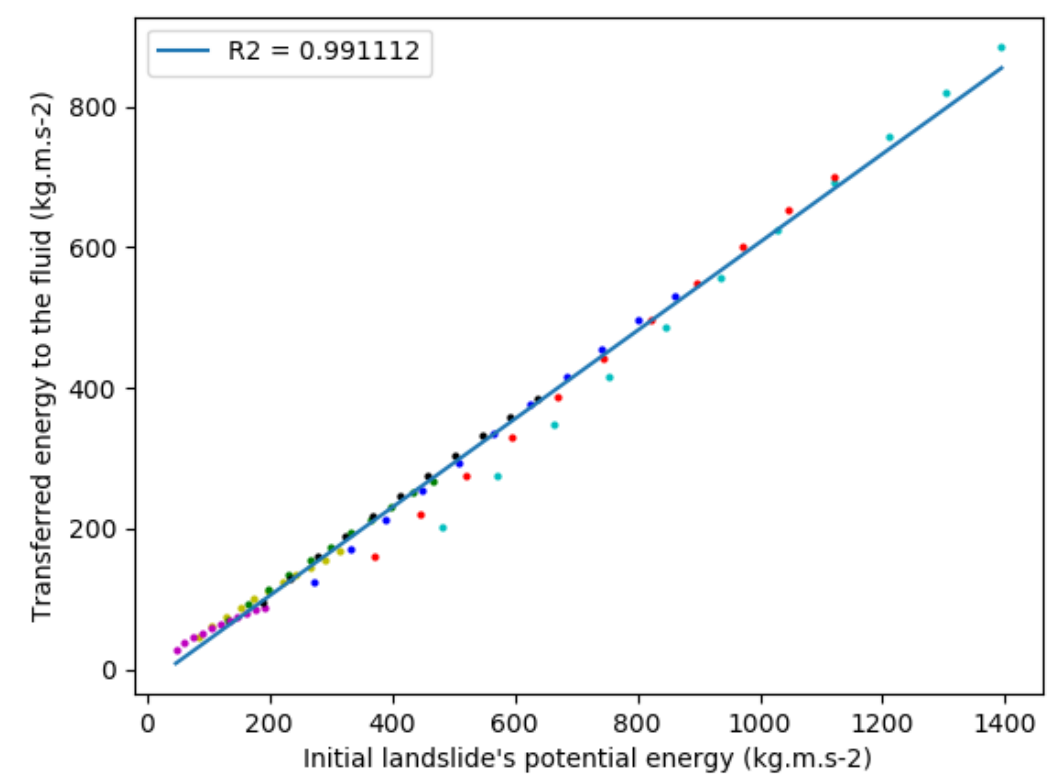

FiguRE 7. Correlation between the initial energy in the landslide and the energy transferred to the water. Each point corresponds to a parameter couple $(H, L)$ while the same color corresponds to the same $H$.
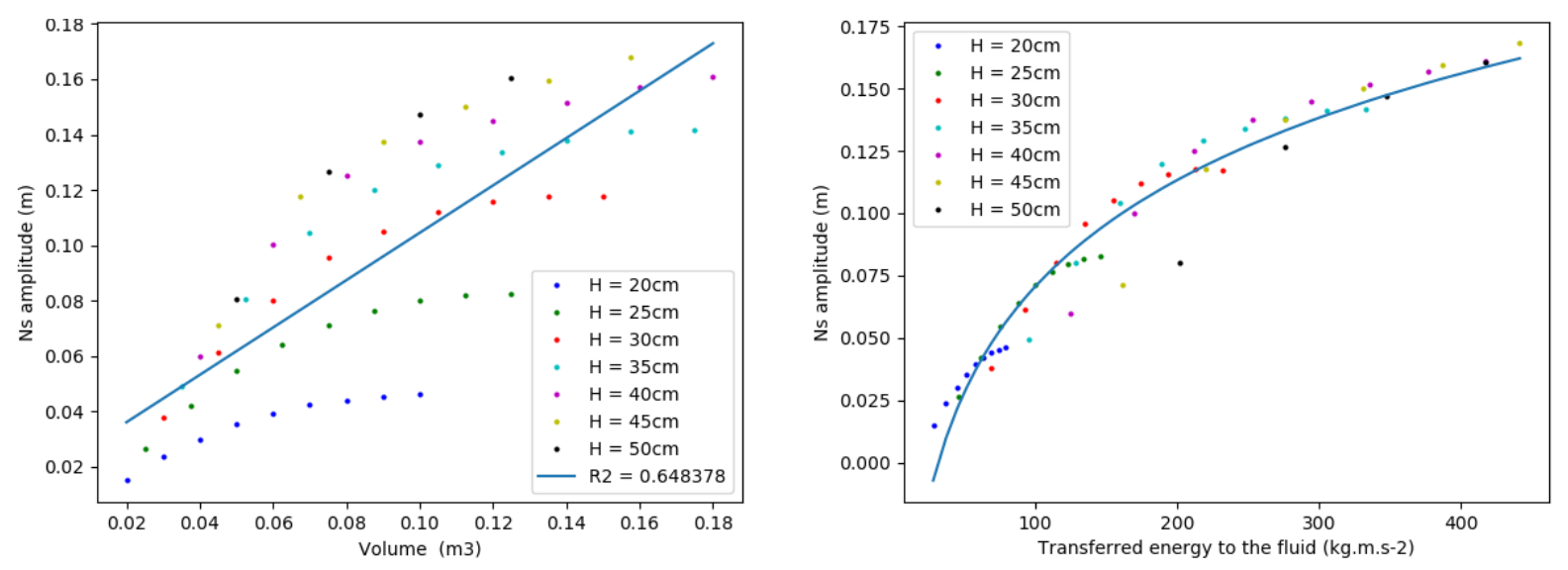

FiguRE 8. Correlation between the amplitude of the wave and respectively the volume of the landslide (left) and the energy transferred to the water (right).

\subsubsection{A shallow water/Boussinesq mixed model}

Based on the observed behaviors of the simplified models described above, we propose a simple mixed model for trying to get results closer to the Navier-Stokes reference solution. Obviously one solution would be to use a fully nonlinear weakly dispersive model, the so called Serre/Green-Naghdi model [14]. In the current state of art of numerical methods, this model is very hard to simulate, in particular close to dry areas. Even with a very robust, entropy-satisfying method [21] we were not able to run the test case.

We propose another strategy using the well validated numerical tools. Since the shallow water model gives a better description of the wave generation process, we propose to use it in the beginning of the simulation, before 

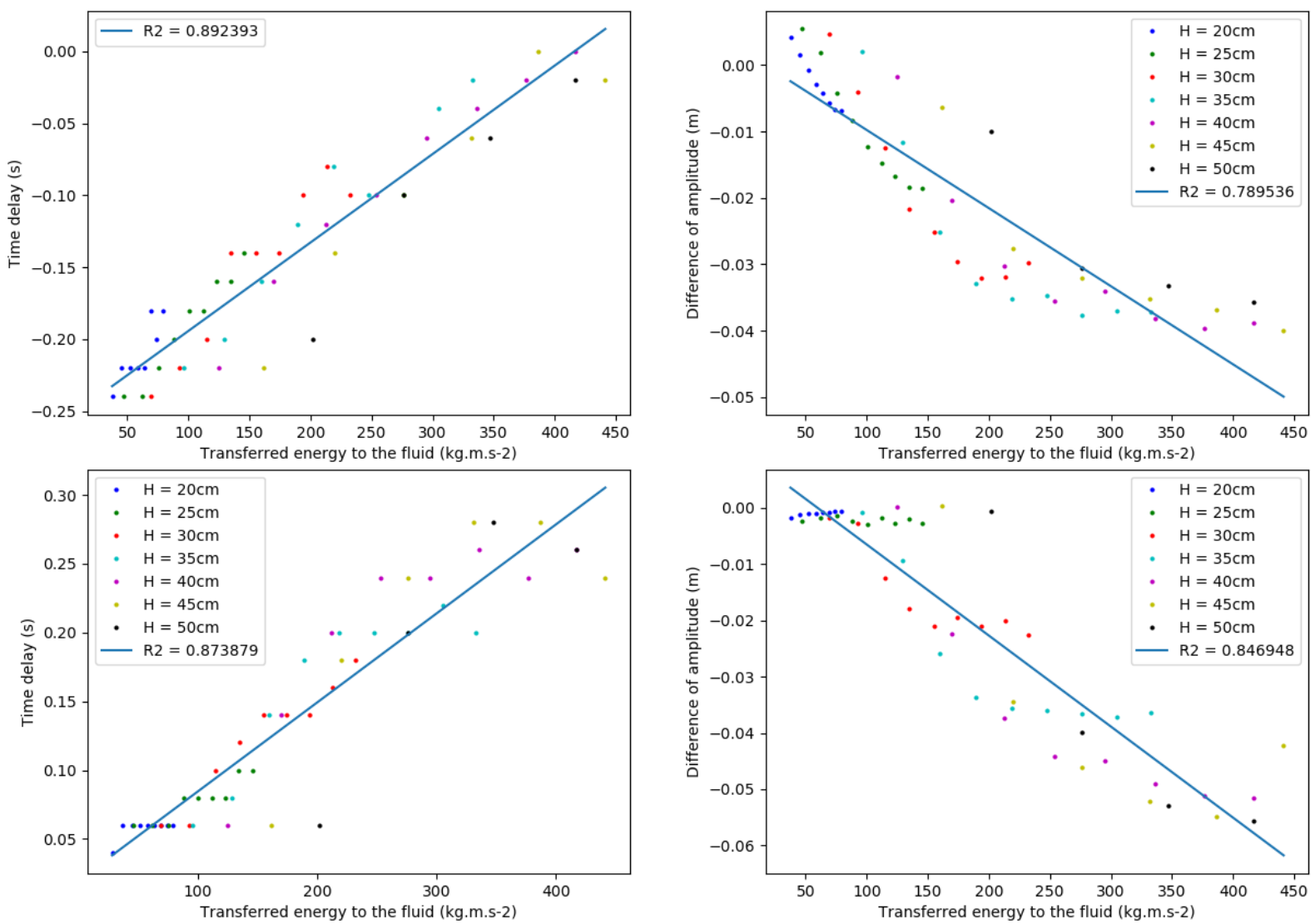

Figure 9. Deviation of the first wave at the chosen probe in term of time delay (left column) and in term of amplitude (right column) of the shallow water model (first line) and the Boussinesq model (second line).

switching to the Boussinesq model after a certain time. In our case where the water is initially at rest, it leads to use the shallow water model during the generation process and the Boussinesq model during the propagation one. However, in a more complex context, as in the case of breaking waves, a space decomposition seems required 20 .

It remains the question of the switch time between models. This time (or localization for space decomposition) can be based on the motion of the bottom. However, to test the idea in this preliminary study, we simply fit it to reproduce as well as possible the reference Navier-Stokes solution. A visual inspection of the simulations points out the approximate instant $t_{\mathrm{switch}}=0.75 \mathrm{~s}$ as a good choice.

In Figure 10, we add the mixed model, i.e. the model where the numerical simulation switches from the shallow water model to the Boussinesq model, to the three fluid mechanics models already presented at the probe $x=2.25 \mathrm{~m}$ (far in the wave propagation zone). Two simulations are presented: in the left graph the ones with the parameters $(H, L)=(0.3 \mathrm{~m}, 0.4 \mathrm{~m})$ corresponding to an intermediary amount of energy, and in the right graph, the ones with the parameters $(H, L)=(0.45 \mathrm{~m}, 0.4 \mathrm{~m})$ corresponding to large amount of energy.

Note that we do not adapt the switch time to the simulations. For both cases, the mixed model leads to a very good agreement with the Navier-Stokes solution in term of time delay of the first wave. The amplitude of the wave with the mixed model is significantly larger when the transferred energy is too big. As far as we understand, this difference can be explained by the fact that for this case the wave energy is large enough for the wave to break in the Navier-Stokes simulation. This wave breaking phenomenon is not present in the Boussinesq model. Some breaking wave strategies existing in the literature can probably improve this result. 

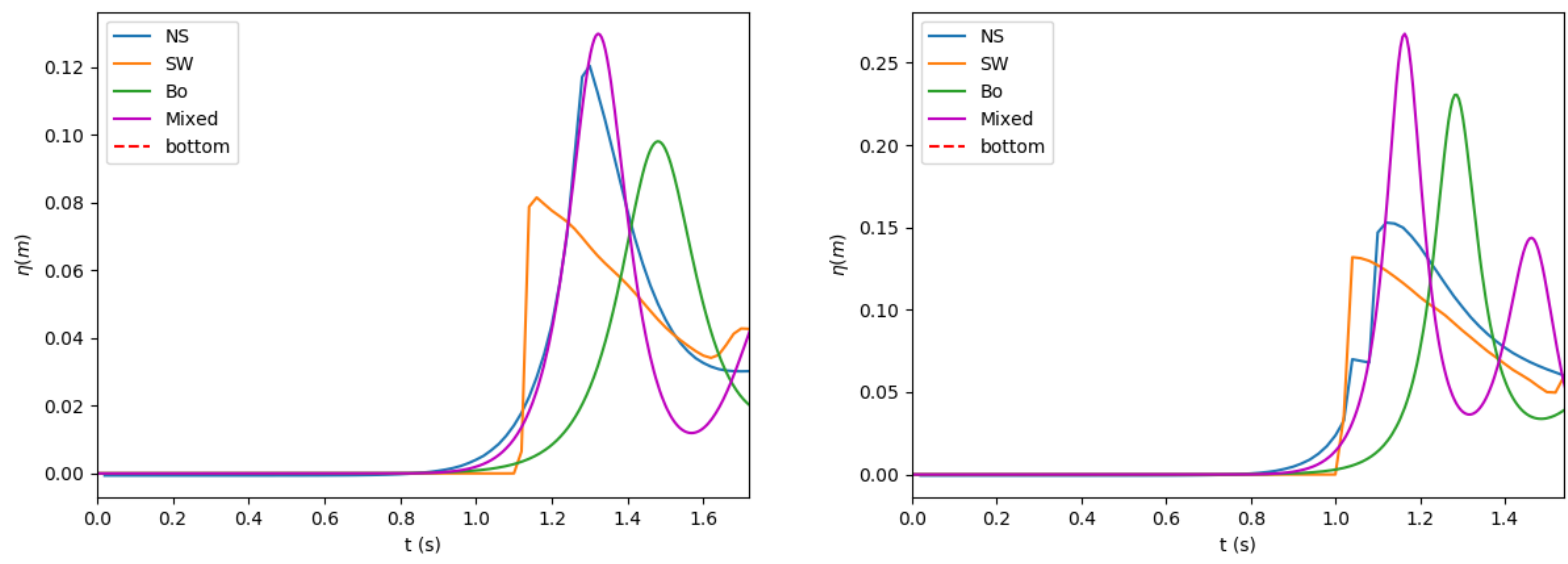

Figure 10. Comparison between the three fluid mechanics models and the proposed mixed shallow water/Boussinesq model, for two test cases at the probe $x=2.25 \mathrm{~m}$.

\section{INVERSE PROBLEM}

The goal of this section is to see if we are able to recover the dynamics of a landslide knowing only the generated waves at some fixed points. This strategy is not trivial for several reasons. First the form of the landslide does not follow a general shape, so we look a priori for a function in an infinite space. Then as we see in the previous section $\$ 3$, even with "exact" landslide shape, the difference between the simplified models and the Navier-Stokes model is significant. Eventually, the solutions of hydrodynamic models can be discontinuous. This kind of solutions are known to be not suitable for inverse problem such as recovering a forcing leading to them [11].

Anyway this preliminary study is performed to observe the result of the strategy in practice. More precisely, the first two points are technical problems that, we hope, could be solved with enough work, while the last one is a theoretical problem that can not be overcame, at least without introducing theoretical tools as Dirac measure that are complex to use in application contexts. However, It happens that computational strategies not confirmed by theory lead to good results.

\subsection{The methodology}

The problem can be summarized by the minimization problem $d=\operatorname{argmin}_{\widetilde{d}}(J(\widetilde{d}))$ associated to the functional

$$
J(\widetilde{d}):=\sum_{i=1}^{N} \int_{0}^{T}\left(\eta\left(x_{i}, t ; \widetilde{d}\right)-\eta_{i}^{\star}(t)\right)^{2} \mathrm{~d} t
$$

where $T$ is the time of observation, $N>0$ is the number of gauges, $x_{i}$ are their position where the observations of the free surface $\eta_{i}^{\star}(t)$ are realized. These observations can be obtained from experiments or from a reference model such as the Navier-Stokes model. The free surface of one of the simplified models $\eta(x, t ; d)(\bar{\eta}=\bar{h}(t, x)-d(t, x)$ for the shallow water model and $\eta$ for the Boussinesq model) depends obviously on the bottom elevation. However, since this dependency is not explicit, the minimization problem has to be solved using numerical tools.

\subsubsection{Modeling assumptions}

As already mentioned, the bottom elevation $d(t, x)$ is a function in an infinite dimensional space (a priori in $L^{1}$ with $\int d(t, x) \mathrm{d} x=\int d(0, x) \mathrm{d} x+B C$ thanks to the mass conservation). The minimization problems in infinite dimensional space are much complex and the numerical resolution is less efficient and less robust than the same ones in finite dimension. To improve the robustness of the strategy, we assume that the bottom can be 
described by a sum of Gaussian functions, leading to a finite number of degrees of freedom. Each Gaussian is characterized by its amplitude $A(t)$, its position $X(t)$ and its standard deviation $\sigma(t)>0$. More precisely we set

$$
\widetilde{d}(t, x)=-\left(B(x)+\sum_{i=1}^{M} A_{i}(t) \exp \left(-\left(\frac{x-X_{i}(t)}{\sigma_{i}(t)}\right)^{2}\right)\right.
$$

where $M>0$ is the number of Gaussians in our approximation, a priori fixed. Assuming the mass conservation for each Gaussian, its standard deviation is linked to its amplitude. More precisely, in one dimensional framework

$$
\begin{aligned}
0 & =\partial_{t}\left(\int_{\mathbb{R}} A_{i}(t) \exp \left(-\left(\frac{x-X_{i}(t)}{\sigma_{i}(t)}\right)^{2}\right) \mathrm{d} x\right) \quad \Rightarrow \quad \sigma_{i}(t)=\frac{A_{i}(0)}{A_{i}(t)} \sigma_{i}(0) . \\
& =\sqrt{\pi} \partial_{t}\left(A_{i}(t) \sigma_{i}(t)\right)
\end{aligned}
$$

It remains to give some assumptions on the form of the time evolution of the Gaussian's amplitude and position. For simplicity of the analysis, we assume a linear evolution:

$$
A_{i}(t)=A_{i}^{0}-a_{i} t \quad \text { and } \quad X_{i}(t)=X_{i}^{0}-c_{i} t
$$

The initial amplitude $A_{i}^{0}$ and position $X_{i}^{0}$ are given by the initial condition, so the only parameters to determine are the decay of amplitude $a_{i} \in \mathbb{R}$ and the velocity of the displacement $c_{i} \in \mathbb{R}$. Using the same strategy, in the case of $M$ Gaussians, we obtain $2 M$ parameters to determine.

\subsubsection{Numerical strategy}

The minimization problem (8) in a finite dimensional space is solved with a very simple gradient descent method [22]. In order to limit the number of direct resolutions, we decouple the resolution of each parameter. More precisely, given a vector of unknown parameters $C$ (in our case $C=\left(a_{i}, c_{i}\right)_{1 \leq i \leq M}$ ) and an initial guess $C_{0}$, we iterate

$$
C_{n+1}=C_{n}-\alpha_{n} \nabla_{C}^{\delta} J\left(C_{n}\right)
$$

where $C_{n}$ is the $\mathrm{n}^{\text {th }}$ approximation of the vector of unknown parameters and $\alpha^{n}$ is the descent step used for the convergence of the method. $\nabla_{C}^{\delta} J$ is an approximation of the gradient $\nabla_{C} J$ of the functional (8). Here we use the finite difference approximation, i.e.

$$
\nabla_{C}^{\delta} J \cdot e_{i}=\frac{J\left(C_{n}+\delta e_{i}\right)-J\left(C_{n}-\delta e_{i}\right)}{2 \delta}
$$

where $\delta$ is the step of the finite difference approximation and $e_{i}$ is the unit vector with all the component vanish except the $i^{\text {th }}$ one. The descent step is $\alpha_{n}$ is chosen to assure that the sequence of approximations applied to the functional is decreasing, i.e. $J\left(C_{n+1}\right)<J\left(C_{n}\right)$. In practice, we consider the sequence defined by

$$
\alpha_{n}^{q+1}= \begin{cases}\omega \alpha_{n}^{q} & \text { if } J\left(C_{n}-\alpha_{n}^{q} \nabla_{C}^{\delta} J\left(C_{n}\right)\right)>J\left(C_{n}\right) \\ \alpha_{n}^{q} & \text { else. }\end{cases}
$$

with $0<\omega<1$. It is clear that the sequence converge and we set $\alpha_{n}=\lim _{q \rightarrow \infty} \alpha_{n}^{q}$.

Note that the estimation of the functional requires one direct computation of a simplified model (6) or (7), and two more for each parameter to estimate the discrete gradient. More efficient methods based on the alternating direction method and the secant approximation can be considered.

\subsection{The numerical results}

In this section, we illustrate the result of the minimization problem for two simple cases. For both simulations, the simplified model used is the shallow water model. The time of observation is set to $t=2 \mathrm{~s}$, and the gauges 

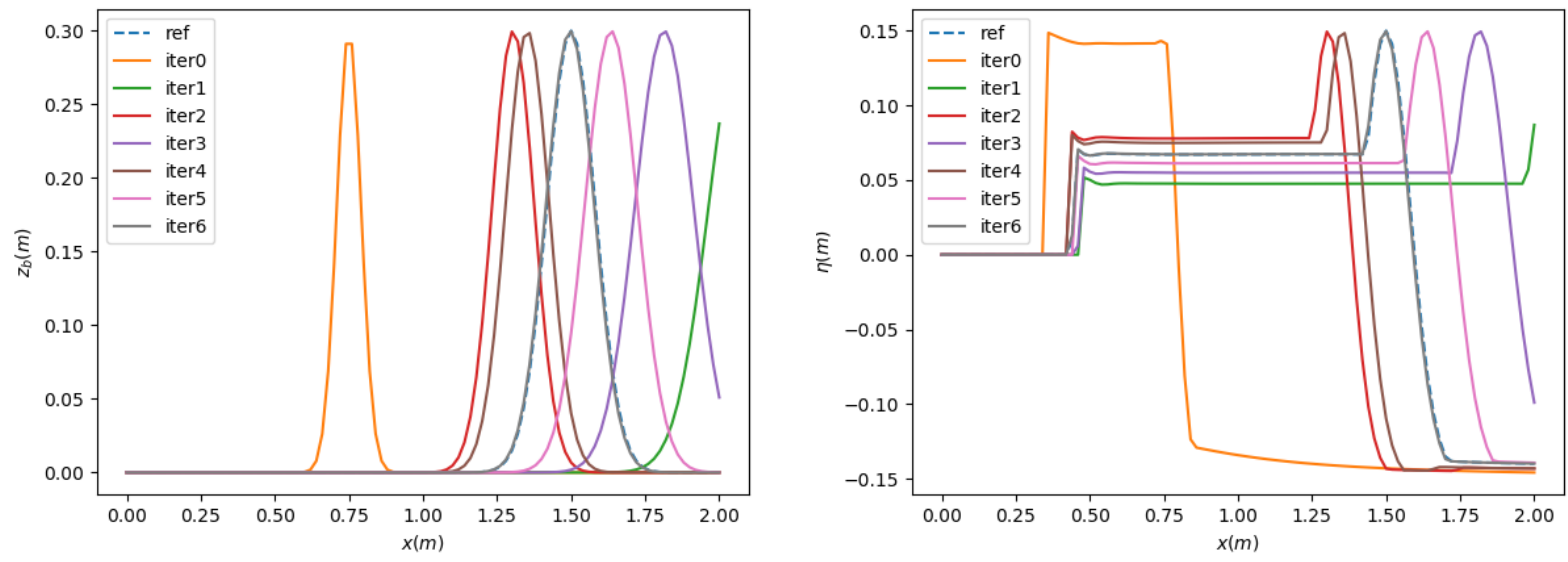

FIGURE 11. Evolution of the bathymetry (left graph) and the generated surface elevation (right graph) along time and iterations for the minimization problem of a Gaussian in translation.
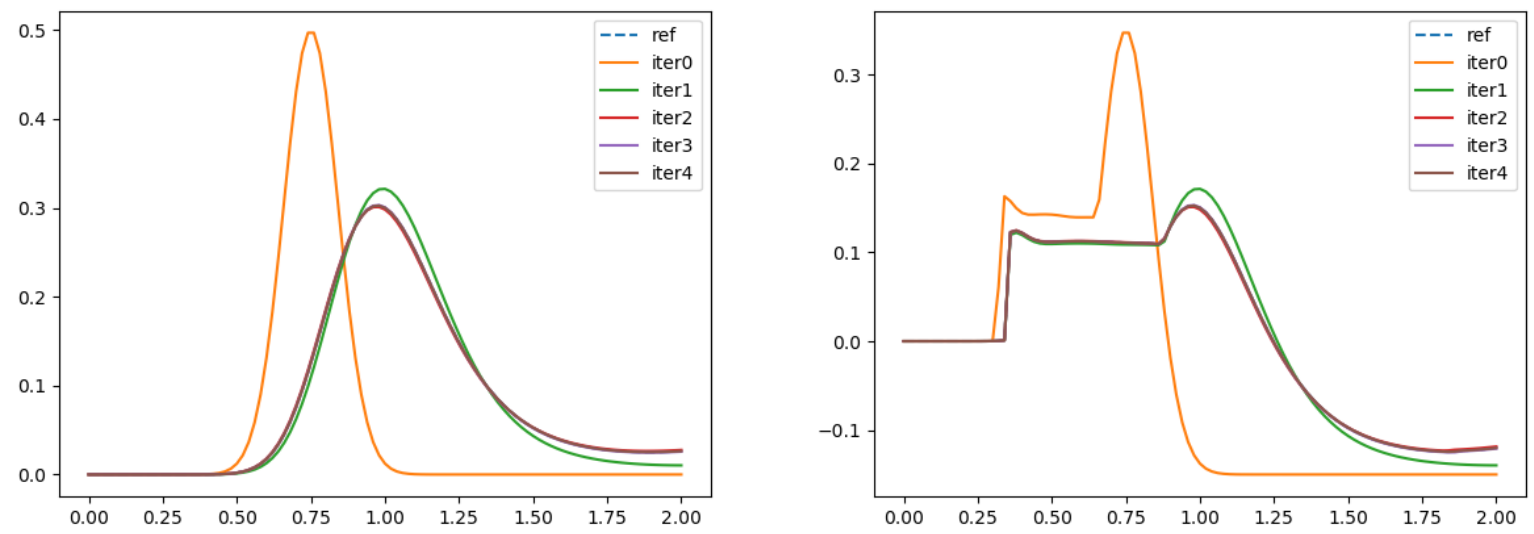

FiguRE 12. Evolution of the bathymetry (left graph) and the generated surface elevation (right graph) along time and iterations for the minimization problem of a Gaussian in translation with amplitude decay.

are located every $0.2 \mathrm{~m}$ from $x=1.4 \mathrm{~m}$ to $x=2.0 \mathrm{~m}$. The observations $\eta_{i}^{\star}$ are obtained by computing the model with the exact solution of the minimization problem (the moving bottom we look for) before forgetting it. The numerical parameters are set by $\delta=10^{-2}$ and $\omega=0.5$ for both simulations.

\subsubsection{Gaussian in translation}

For this test case, we consider only one Gaussian $M=1$ that does not decay in amplitude during the propagation, i.e. $a_{1}=0$. The only parameter to determine is $c_{1}$ and it is set to 0.5 to realize the observations.

In Figure 11, the reference solution and the solution obtained along the iterations of the gradient descent method are plotted. We recover the velocity of the Gaussian with few iterations.

\subsubsection{Gaussian in translation with decay}

For this test case, we still consider only one Gaussian $M=1$ but with a linear decay in amplitude. Two parameters have to be determined, i.e. $\left(a_{1}, c_{1}\right)$ and it is set to $(-0.2,0.75)$ to realize the observations. 
In Figure 12, the reference solution and the solution obtained along the iterations of the gradient descent method are plotted. We recover the velocity of the Gaussian with few iterations.

In both cases the minimization strategy converges in few iterations to the solution we look for. However, other numerical tests must be performed before concluding that the strategy can be used in an application context.

\section{CONCLUSiOn}

In this paper, we analysed the relevance of the use of the shallow water model or the Boussinesq model to simulate tsunamis generated by a landslide. Two questions were considered.

First we compared the two models to a reference solution obtained using the Navier-Stokes model. This comparison highlighted the fact that the Boussinesq model is not able to represent the wave generated in the wave generation zone. For large enough landslide, even in the wave propagation zone (at least not too far from the wave generation zone), the shallow water model is more efficient. We propose a strategy based on a switch from the shallow water model during the generation of the wave to the Boussinesq model during the propagation of the wave. This strategy seems relevant to improve the results of the simulations. Another solution, more satisfying in theory, is the application of more sophisticated models, as the Serre/Green-Naghdi model. However, it seems that more robust schemes, in particular for moving bathymetry or stiff solution, are required.

In addition, we highlighted the fact that the landslide is not well characterized by the volume of the landslide but can be better characterized by the energy transferred to the water. This energy is well correlated to the initial potential energy of the landslide that can be determined by the initial condition.

Finally, we addressed the question of the possibility of determining the landslide from the generated wave. It was formulated as an minimization problem, in which we searched the time-dependent bottom that minimizes the difference between a reference surface elevation and the computed surface elevation obtained by the bottom to be determined. The question was brought back to a limiting number of unknowns to determine by assuming that the bottom can be well represented by a finite (small) number of Gaussians with parameters following some empirical laws. Even if our computational analysis was too limited to conclude, the numerical strategy was validated in simple cases. Deeper computational analysis is however required, in particular a crucial point will be to determine if the sum of Gaussians is able to well approximate a realistic landslide by the inverse problem.

\section{REFERENCES}

[1] P. Heinrich and A. Piatanesi, "Near-field modeling of the July 17, 1998 tsunami in Papua New Guinea," Geophysical Research Letters, vol. 27, no. 19, pp. 3037-3040, 2000.

[2] H. Hébert, A. Piatanesi, P. Heinrich, and F. Schindelé, "Numerical modeling of the September 13, 1999 landslide and tsunami on Fatu Hiva Island (French Polynesia)," Geophysical Research Letters, vol. 29, no. 10, pp. 10-13, 2002.

[3] A. Le Friant, P. Heinrich, C. Deplus, and G. Boudon, "Numerical simulation of the last flank-collapse event of Montagne Pelée, Martinique, Lesser Antilles," Geophysical Research Letters, vol. 30, no. 2, 2003.

[4] M. Rodriguez, N. Chamot-Rooke, H. Hébert, M. Fournier, and P. Huchon, "Owen Ridge deep-water submarine landslides: Implications for tsunami hazard along the Oman coast," Natural Hazards and Earth System Science, vol. 13, pp. 417-424, 2013.

[5] A. Poupardin, P. Heinrich, A. Frère, D. Imbert, H. Hébert, and M. Flouzat, "The 1979 Submarine Landslide-Generated Tsunami in Mururoa, French Polynesia," Pure and Applied Geophysics, vol. 174, pp. 3293-3311, 2017.

[6] A. Paris, E. Okal, C. Guérin, P. Heinrich, F. Schindelé, and H. Hébert, "Numerical modeling of the June 17, 2017 landslide and tsunami events in Karrat Fjord, west Greenland," Pure and Applied Geophysics, vol. 176, no. 7, pp. 3035-3057, 2019a.

[7] A. Paris, P. Heinrich, R. Paris, and S. Abadie, "The December 22, 2018 Anak Krakatau, Indonesia, landslide and tsunami: preliminary modeling results," Pure and Applied Geophysics, 2019b.

[8] S. Le Roy, D. Monfort, and R. Pedreros, Projet ALDES : Simulations numériques de tsunamis en Méditerranée Occidentale. Rapport BRGM/RP-61473-FR, 101 p., 62 ill., 2012.

[9] P. Watts, S. T. Grilli, J. T. Kirby, G. J. Fryer, and D. R. Tappin, "Landslide tsunami case studies using a Boussinesq model and a fully nonlinear tsunami generation model," Natural Hazards and Earth System Science, vol. 3, no. 5, pp. 391-402, 2003.

[10] S. T. Grilli and P. Watts, "Tsunami generation by submarine mass failure. i: Modeling, experimental validation, and sensitivity analyses," Journal of Waterway, Port, Coastal, and Ocean Engineering, vol. 131, no. 6, pp. 283-297, 2005.

[11] C. Fiorini, C. Chalons, and R. Duvigneau, "A modified sensitivity equation method for the Euler equations in presence of shocks," Numerical Methods for Partial Differential Equations, 2019. 
[12] S. Viroulet, A. Sauret, and O. Kimmoun, "Tsunami generated by a granular collapse down a rough inclined plane," EPL (Europhysics Letters), vol. 105, p. 34004, feb 2014.

[13] OpenCFD Ltd.: OpenFOAM - The Open Source CFD Toolbox - User Guide, 2004. https://www.openfoam.com/documentation/user-guide/.

[14] D. Lannes, The water waves problem : mathematical analysis and asymptotics, vol. 188. Mathematical Surveys and Monographs, 2013.

[15] F. Bouchut, Nonlinear stability of finite volume methods for hyperbolic conservation laws, and well-balanced schemes for sources. Springer Science \& Business Media, 2004.

[16] E. Audusse, F. Bouchut, M.-O. Bristeau, R. Klein, and B. Perthame, "A fast and stable well-balanced scheme with hydrostatic reconstruction for shallow water flows," SIAM J. Sci. Comput., vol. 25, no. 6, pp. 2050-2065, 2004.

[17] F. Løvholt, G. Pedersen, and G. Gisler, "Oceanic propagation of a potential tsunami from the La Palma Island," Journal of Geophysical Research: Oceans, vol. 113, no. 9, pp. 1-21, 2008.

[18] Y. Zech, S. Soares-Frazão, B. Spinewine, C. Savary, and L. Goutière, "Inertia effects in bed-load transport models," Canadian Journal of Civil Engineering, vol. 36, no. 10, pp. 1587-1597, 2009.

[19] Fernández-Nieto, Enrique D., Luna, Tomás Morales de, Narbona-Reina, Gladys, and Zabsonré, Jean de Dieu, "Formal deduction of the saint-venant-exner model including arbitrarily sloping sediment beds and associated energy," ESAIM: M2AN, vol. 51, no. 1, pp. 115-145, 2017.

[20] A. Filippini, M. Kazolea, and M. Ricchiuto, "A flexible genuinely nonlinear approach for nonlinear wave propagation, breaking and run-up," Journal of Computational Physics, vol. 310, pp. $381-417,2016$.

[21] M. Parisot, "Entropy-satisfying scheme for a hierarchy of dispersive reduced models of free surface flow," International Journal for Numerical Methods in Fluids, vol. 91, no. 10, pp. 509-531, 2019.

[22] J. Nocedal and S. J. Wright, Numerical Optimization. Springer, 2nd ed., 2006. 